\title{
A group selection approach to supplier collaborative configuration problems with correlation of experts and attributes
}

\author{
Haihan Chen \\ School of Economics and Management, Fuzhou University, Fuzhou, Fujian, 350116, China
}

Received 23 April 2015

Accepted 25 August 2015

\begin{abstract}
Enterprises nowadays are often confronted with selecting supplier collaborative configuration problems in supply chain management. The aim of this paper is to develop a group selection methodology with the correlation of experts and attributes for solving supplier collaborative configuration problems. In this methodology, opinions of experts for supplier collaborative configuration on the basic attributes are integrated, respectively. The Denoeux cautious combination rules are further modified to delete the ignorance caused by relative weights in the aggregation and hereby we estimate the group evaluations of supplier collaborative configuration, which are used to rank supplier collaborative configuration schemes/alternatives through employing the minimum/maximum regret value method. A real case is used to verify and show the rationality, effectiveness and applicability.
\end{abstract}

Keywords: Supplier collaborative configuration; Group decision making; Decision analysis; Multiattribute decision making; Evidential reasoning

\section{Introduction}

In recent years, the increasing complexity of components and parts has raised the development costs of new products and development uncertainty. Therefore, a more emphasis on value creation has spurred substantial interest in the concept of cost control in complex environments in supply chains ${ }^{1-4}$. In the recent survey, $\mathrm{Su}^{5}$ proposed a hierarchical grey decision-making trial and evaluation laboratory method to identify and analyze criteria and alternatives in incomplete information. Su's method may identify supplier prioritization. Verdouw ${ }^{6}$ proposed a control model for object virtualization in supply chain management to define feasible options for the virtualization of supply chain control. In the innovation-driven times, people pay more attention to collaboration and believe that enterprises should collaborate with suppliers to complete the development and manufacturing tasks of new products. The collaboration could be more efficient and create more social value. During the new product development process, the supplier collaborative management significantly affects the quality, price and delivery date of the final products. Hence, it is necessary to study collaborative relationships between enterprises and suppliers, which may help enterprises collaboratively configure suppliers in a scientific and rational manner. Consequently, the production costs of the enterprises are further reduced and hereby the profits are enhanced. This paper will focus on developing effective and practical group selection models and method of the supplier collaborative configuration problems.

Recently, there are few of articles involved in the supplier collaborative configuration problems ${ }^{7-8}$. Fuzzy DEMATEL technique ${ }^{9-10}$, evidential reasoning method $^{11-12}$ and hybrid solution ${ }^{13-14}$ were widely used. Therefore, the evidential reasoning theory with the advantages of dealing with uncertainty was more adaptive to the supply chain management. For instance, the traditional evidential reasoning method was used to solve the design and contractor selection problems of complex engineering products ${ }^{15-16}$. The rules and utility 
of evidential reasoning were used to deal with the uncertainty problems of attributes in multi-attribute decision analysis ${ }^{17-19}$. The evidential reasoning method was applied to multi-attribute decision analysis with uncertain intervals, which has incomplete information or total ignorance ${ }^{20}$. Ölcer ${ }^{21}$ explored a new fuzzy multi-attribute decision-making method, which is used to solve the aggregation problems of fuzzy qualitative attributes and the fuzzy multi-attribute group decision-making problems. $\mathrm{Fu}$ and $\mathrm{Fu}^{22-23}$ proposed a new system that integrates multi-agents, context-aware computing, and context-aware reasoning or case-based reasoning to improve cost collaborative management in supply chains. The preceding works were primarily based on evidential reasoning in which the aggregation problem of fuzzy attributes is a significant and difficult aspect in decision analysis.

For supplier collaborative configuration selection problems, Dickson ${ }^{24}$ chose 23 fuzzy and non-fuzzy evaluation criteria. Combining the ANP with multi-objective linear programming, Demirtas ${ }^{25}$ developed a method for selecting suppliers. $\mathrm{Li}^{26}$ regarded outsourcing provider selection problems as a type of fuzzy inhomogenous multi-attribute group decision making problems, which are solved through developing a new fuzzy mathematical programming method. $\mathrm{Hu}^{27}$ studied the supplier selection problems in green procurement under the mode of low carbon economy. Zhang ${ }^{28}$ adopted a framework of a central coordination system, which is equipped with a multi-criteria genetic optimization feature, to reduce less complexity in the evaluation stage. As far as we know, although there are some researches on the supplier collaborative configuration problems, but most of them have limited application situations for mathematic constraints. Therefore, the aim of this paper is to develop new group selection models and method for solving supplier collaborative configuration problems based on the correlation of experts and attributes in which there are cognitive uncertainties of experts from the purchasing, quality, research and development (R\&D), and sales departments of the enterprise. Compared with the existing related methods, the method proposed in this paper has three innovation aspects. Firstly, we loosen the constraints of the independence of expert opinions, which allow experts to share their experience and knowledge. Secondly, the selection process of the supplier collaborative configuration problems coincides with reality. Second, the difficulty of attribute selection is reduced, and the dominant or recessive correlation between partial attributes cannot be repelled by loosening the independence requirement. As a result, the robustness of optimal selection results of the supplier collaborative configuration problems is enhanced. Thirdly, the developed method is of more extensive applications due to adequately modifying the Denoeux cautious combination rules ${ }^{29}$.

\section{Some concepts and notations}

For convenience to understand the group selection methodology with the correlation of experts and attributes, we briefly review some relevant concepts and notations.

\subsection{Belief functions and evidential reasoning}

The belief function theory is a theoretical framework for evidential reasoning with partial and unreliable information $^{20,30-31}$. Let a finite set $\Omega$ be the frame of discernment and $S$ be the evidential source on $\Omega$. Partial knowledge regarding the actual value of $S$ taken by the variable $\omega$ is represented by a belief function, which is defined as a function $m$ from $2^{\Omega}$ to $[0,1]$, so that:

$$
\sum_{\mathbf{A} \subseteq \Omega} m(A)=1
$$

where $m(A)>0$ and each focal set $A$ is a set of possible values for $\omega$. The quantity $m(A)$ can be interpreted as the measure of beliefs, which is committed exactly to $\omega \in A$ on the basis of a given evidential corpus. Complete ignorance corresponds to $m(\Omega)=1$, whereas perfect knowledge of the value of $\omega$ is represented by the allocation of the whole mass of beliefs to a unique singleton of $\Omega$. The belief function with nested focal elements is said to be consonant. $m$ is said to be simple support if there exist $A \subset \Omega$ and $w \in[0,1]$ so that $m(A)=1-w$ and $m(\Omega)=w$, and all other masses are equal to zero. If $m(\Phi)=0$, where $\Phi$ is an empty set, then $m$ is said to be normal. The belief function $m$ can be represented by a commonality function with $q(A)=\sum_{B \supseteq A} m(B)$ for any $A \subseteq \Omega$. For decomposable and orthodox belief function $m$, it can be defined as the following standard combination of several simple belief functions: 


$$
m=\oplus_{\phi \neq A \subset \Omega} A^{w(A)}
$$

where $w(A)$ is defined as a weight function for any $A \subset \Omega$ and $A \neq \Phi, w(A) \in[0,1]$. The symbol $\oplus$ is defined as a Dempster-Shafer composition operator. Extendedly, for any unorthodox belief function $m$, it cannot be expressed as Eq. (3), thereof any $A \subset \Omega$ and $w(A) \in[0,+\infty)$. The extended $w(A)$ is defined as Eq. (4), thereof $2 N$ is denoted as even number set and $|A|$ is denoted as the cardinality of $A$.

$$
w(A)=\prod_{B \supseteq A} q(B)^{(-1)|B|-|A|+1}=\left\{\begin{array}{l}
\frac{\prod_{B \supseteq A,|B| \notin 2} q(B)}{\prod_{B \supseteq A,|B| \in 2} q(B)},|A| \in 2 N \\
\frac{\prod_{B \supseteq A,|B| \in 2} q(B)}{\prod_{B \supseteq A,|B| \notin 2} q(B)},|A| \notin 2 N
\end{array}\right.
$$

which can be simplified as follows:

$$
w(A)=\left\{\begin{array}{l}
\frac{m(\Omega)}{m\left(h_{p}\right)+m(\Omega)}, A=h_{p} \\
m(\Omega) \Pi_{p=1}^{p}\left[1+\frac{m\left(h_{p}\right)}{m(\Omega)}\right], A=\phi \\
1 \quad \text { otherwise }
\end{array}\right.
$$

When the reliability of a source is doubtful, the mass provided by this source can be discounted through using Eq. (6):

$$
\left\{\begin{array}{l}
m^{\lambda}(A)=(1-\lambda) m(A) \\
m^{k}(\Omega)=(1-\lambda) m(\Omega)+\lambda
\end{array} \quad A \neq \Omega\right.
$$

where $\lambda \in\lceil 0,1\rceil$ is the discount rate. The discount rate is related to the confidence held by an external agent in the reliability of the source. It can be interpreted as the plausibility that the source is unreliable. When $\lambda$ is equal to 1 , the vacuous mass function is obtained. When $\lambda=0, m$ remains unchanged.

\subsection{Denoeux synthetized rules}

Assume that $m_{1}$ and $m_{2}$ are two dependent belief functions which are induced by distinct items of evidences on $\Omega$. The Denoeux synthetized rules $^{29}, 32$ can be defined as follows:

$$
\begin{aligned}
& m_{1 \wedge 2}=m_{1} \wedge m_{2}=\underset{\substack{\Phi \neq A \subset \Omega \\
\oplus}}{\oplus w_{1 \wedge 2}{ }^{(A)}} \\
& w_{1 \wedge 2}(A)=\min \left\{w_{1}(A), w_{2}(A)\right\}
\end{aligned}
$$

where $w_{1 \wedge 2}(A)$ is a conjunction weight, and the operator " $\Lambda$ " has the characteristics of interactivity, conjunction and power.
Considering the ignorance caused by relative weight or discount and incomplete information, the modified Denoeux cautiously synthesized rules are given as follows:

$$
\begin{gathered}
m_{1 \wedge} \wedge^{*}=m_{1} \wedge^{*} m_{2}=\underset{\Phi \neq A \subset \Omega}{\oplus} A^{w_{1 \wedge 2}{ }^{(A)}} \\
w_{1 \wedge{ }^{*} 2}(A)=\min \left\{\begin{array}{lr}
\min \left\{w_{1}(A), w_{2}(A)\right\}, A=h_{p}, p=1,2, \ldots, P \\
\min \left\{w_{1}(A), w_{2}(A)\right\}, & A=\phi \\
\min \left\{w_{1}(A), w_{2}(A)\right\}, & A=\bar{\Omega}
\end{array}\right.
\end{gathered}
$$

where $\Omega=\left\{h_{p} \mid p=1,2, \ldots, P\right\}$, and $\bar{\Omega}$ is an ignorance set.

\section{Representation of supplier collaboration configuration problems}

Assume that there are $M$ supplier collaboration configuration schemes, which are respectively denoted by $A_{l}(l=1,2, \ldots, M) . \quad L$ basic attributes are respectively denoted by $e_{i}(i=1,2, \ldots, L)$. The weight vector of the basic attributes is denoted by $w=\left(w_{1}, w_{2}, \ldots, w_{L}\right)$, where $0 \leq w_{i} \leq 1$ $(i=1,2, \ldots, L)$ and $\sum_{i=1}^{L} w_{i}=1$.

Meanwhile, $T$ experts are denoted by $t_{k}(k=1,2, \ldots, T)$. The relative weight vector of $T$ experts is denoted by $\lambda$ $=\left(\lambda_{1}, \lambda_{2}, \ldots, \lambda_{T}\right)$, where $0 \leq \lambda_{k} \quad \leq 1 \quad(\quad k=1,2, \ldots, T)$ and $\sum_{k=1}^{T} \lambda_{k}=1$.

The experts evaluate the $M$ supplier collaboration configuration schemes on $L$ basic attributes and hereby form the set of evaluation ratings with mutual exclusive and complete characteristics. The evaluation ratings are expressed as the set $\Omega=\left\{H_{n} \mid n=1,2, \ldots, N\right\}$. Moreover, let $S_{i}^{k}\left(A_{l}\right)=\left\{\left(H_{n}, \beta_{n, i}^{k}\left(A_{l}\right)\right) \mid n=1,2, \ldots, N\right\}$ represent the distributed evaluation set of the expert $t_{k}(k=1,2, \ldots, T)$ for the supplier collaboration configuration scheme $A_{l}(l=1,2, \ldots, M)$ on the attribute $e_{i}(i=1,2, \ldots, L)$. The belief degrees of the evaluation set on the evaluation rating $H_{n}$ are $\beta_{n, i}^{k} \quad\left(A_{l}\right)$, where $\beta_{n, i}^{k} \quad\left(A_{l}\right) \geq 0$ and $\sum_{n=1}^{N} \beta_{n, i}^{k}\left(A_{l}\right) \leq 1$. When $\sum_{n=1}^{N} \beta_{n, i}^{k}\left(A_{l}\right)=1$, the distributed evaluation set is complete; otherwise, it is incomplete. If the distributed evaluation set is incomplete, then the unallocated belief degree is assigned to $\Omega$. That is, $\quad \sum_{n=1}^{N} \beta_{n, i}^{k}\left(A_{l}\right)+\beta_{\Omega, i}^{k} \quad\left(A_{l}\right)=1 . \quad$ In particular, $\sum_{n=1}^{N} \beta_{n, i}^{k}\left(A_{l}\right)=0$ represents complete ignorance. Once $M$ supplier collaboration configuration schemes are evaluated on $L$ basic attributes, we can obtain the belief decision matrix as follows: 


$$
D_{g}^{k}=\left(S_{i}^{k}\left(A_{l}\right)\right)_{L \times M}
$$

\section{Group selection models and method of supplier collaboration configuration problems}

\subsection{Aggregation method of expert opinions on basic attributes}

\subsubsection{Determination of relative weights}

The relative weights of the group opinions of experts $t_{k}(k=1,2, \ldots, T)$ for the supplier collaboration configuration schemes $A_{l}(1 \leq l \leq M)$ on basic attributes $e_{i}$ $(1 \leq i \leq L)$ can be computed by using the following formulae (i.e., Eqs. (12)-(17)):

$$
\begin{aligned}
& m_{n, i}^{k}=m_{i}^{k}\left(H_{n}\right)=\lambda_{k} \beta_{n, i}^{k}\left(A_{l}\right) \\
& m_{\Omega, i}^{k}=m_{i}^{k}(\Omega)=1-\lambda_{k} \sum_{n=1}^{N} \beta_{n, i}^{k}\left(A_{l}\right) \\
& \bar{m}_{\Omega, i}^{k}=\bar{m}_{i}^{k}(\Omega)=1-\lambda_{k} \\
& \tilde{m}_{\Omega, i}^{k}=\tilde{m}_{\Omega, i}^{k}(\Omega)=\lambda_{k}\left(1-\sum_{n=1}^{N} \beta_{n, i}^{k}\left(A_{l}\right)\right) \\
& m_{\Omega, i}^{k}=\bar{m}_{\Omega, i}^{k}+\tilde{m}_{\Omega, i}^{k} \\
& \sum_{k=1}^{T} \lambda_{k}=1
\end{aligned}
$$

Thus, the opinion of the expert $t_{k}$ for the supplier collaboration configuration scheme $A_{l}$ on the basic attribute $e_{i}$ can be expressed as $m_{i}^{k}=\left\{\left(H_{1}, m_{1, i}^{k}\right),\left(H_{2}, m_{2, i}^{k}\right), \ldots,\left(H_{N}, m_{N, i}^{k}\right),\left(\Omega, m_{\Omega, i}^{k}\right)\right\}$.

In the aforementioned relative weight determination, the ignorance $m_{\Omega, i}^{k}$ is divided into $\bar{m}_{\Omega, i}^{k}$ and $\tilde{m}_{\Omega, i}^{k}$, which are respectively caused by the relative weight of the basic attribute $e_{i}$ and the incompleteness that the basic attribute $e_{i}$ evaluates $A_{l}$. In this paper, we use the analytical synthesis method ${ }^{20}$ to deal with the two parts of ignorance differently. $\bar{m}_{\Omega, i}^{k}$ has no relationship with the expert opinions and is only used to deal with the conflicts between the expert opinions. Therefore, $\bar{m}_{\Omega, i}^{k}$ is deleted from the synthesized results and only maintains $\tilde{m}_{\Omega, i}^{k}$. In the following group opinion aggregation, $\bar{m}_{\Omega . i}^{k}$ is also deleted according to the modified Denoeux relevant belief synthesized rules.

\subsubsection{Aggregation of expert opinions on the basic attributes}

In this subsection, let us consider expert opinions involving ignorance caused by the relative weights. However, those opinions cannot be rationally synthesized by the Denoeux cautiously synthesized rules ${ }^{29}$ because such ignorance cannot be deleted. Therefore, the Denoeux cautiously synthesized rules should be modified to facilitate the involvement of such ignorance in the aggregation of expert opinions and subsequently delete the ignorance from the synthesized results.

We use the example of expert group opinions for the supplier collaboration configuration schemes $A_{l}(1 \leq l \leq M)$ on the basic attributes $e_{i}(1 \leq i \leq L)$ after processing by $\lambda_{k}$ to elaborate the modified Denoeux cautiously synthesized rules and explain the aggregation process of expert group opinions.

(1) Weight functions of the ignorance caused by the relative weights

Denoeux ${ }^{29}$ argued that an arbitrary non-dogmatic belief function can be divided into a series of simple belief functions and the corresponding weight function $w(A) \in[0,+\infty)$ can be defined as $w(A)=\prod_{B \supset A} q(B)^{(-1)^{|B|-|A|+1}}$. Apparently, the function $w(A)$ does not involve the ignorance caused by the relative weights. Given that the ignorance caused by the relative weights should be deleted in the aggregation of expert opinions, the weight function should be extended to represent such ignorance. Generally, for an arbitrary expert's opinion $m$ which is processed by the relative weights, it follows that $m(\Omega)=m(\bar{\Omega})+m(\widetilde{\Omega})$, where $m(\bar{\Omega})$ denotes the ignorance caused by the incompleteness of the expert opinion $m$, and the relative weight of ignorance can be computed as follows:

$$
w(\bar{\Omega})=\frac{m(\widetilde{\Omega})}{m(\widetilde{\Omega})+m(\bar{\Omega})}
$$

Based on the preceding results, for any $A \subset \Omega$ or $A=\bar{\Omega}$, the nonstandard decomposition of the expert opinion $m$ is expanded as follows:

where $w(A) \in[0,+\infty)$.

$$
m=\oplus_{A \subset \Omega} A^{w(A)}
$$

According to Eq. (18), weights of the opinions $m_{i}^{k}$ from the expert $t_{k}$ on the basic attribute $e_{i}$ are computed as follows:

$$
w_{i}^{k}(\bar{\Omega})=\frac{\tilde{m}_{\Omega, i}^{k}}{\bar{m}_{\Omega, i}^{k}+\widetilde{m}_{\Omega, i}^{k}}
$$

(2) Weight functions of expert opinions

As a result, according to Eq. (18), combining with Eq. (20), the weight functions of expert opinions $m_{i}^{k}$ are calculated by using the following formulae (i.e., Eqs. (21)-(23)): 


$$
\begin{gathered}
\{\varnothing\}: w_{i}^{k}(\varnothing)=m_{\Omega, i}^{k} \prod_{n=1}^{N}\left(1+\frac{m_{n, i}^{k}}{m_{\Omega, i}^{k}}\right) \\
\left\{H_{n}\right\}: w_{i}^{k}\left(H_{n}\right)=\frac{m_{\Omega, i}^{k}}{m_{n, i}^{k}+m_{\Omega, i}^{k}} \\
\{\bar{\Omega}\}: w_{i}^{k}(\bar{\Omega})=\frac{\tilde{m}_{\Omega, i}^{k}}{\bar{m}_{\Omega, i}^{k}+\widetilde{m}_{\Omega, i}^{k}}
\end{gathered}
$$

(3) Synthesis of the weight functions of expert group opinions

Once the weight functions $w_{i}^{k}$ of $m_{i}^{k}$ are synthesized, then we can compute the weight functions of expert group opinion for the supplier collaboration configuration schemes $A_{l}(1 \leq l \leq M)$ on the basic attributes $e_{i}(1 \leq i \leq L)$ according to the following formulae (i.e., Eqs. (24)-(26)):

$$
\begin{gathered}
\{\varnothing\}: w_{i}(\varnothing)=\min \left\{w_{i}^{k}(\varnothing) \mid k=1,2, \ldots, T\right\} \\
\left\{H_{n}\right\}: w_{i}\left(H_{n}\right)=\min \left\{w_{i}^{k}\left(H_{n}\right) \mid k=1,2, \ldots, T\right\} \\
\{\bar{\Omega}\}: w_{i}(\bar{\Omega})=\min \left\{w_{i}^{k}(\bar{\Omega}) \mid k=1,2, \ldots, T\right\}
\end{gathered}
$$

(4) Aggregation of expert group opinions for schemes on basic attributes

Using Eqs. (24)-(26), the standardized belief function can be computed as follows:

$$
m_{i}=\theta \oplus_{\varnothing \neq A \subset \Omega} A^{w_{i}(A)}
$$

where the standardized

coefficient is $\theta$ and $\theta=\left(1-m_{i}(\varnothing)-m_{i}(\bar{\Omega})\right)^{-1} \cdot m_{i} \bar{\Omega}()$ is introduced to delete the ignorance so that it ensures the rationality of the synthesized results.

\subsection{Aggregation of expert opinions on supplier collaboration configuration schemes}

Aggregation of expert opinions on supplier collaboration configuration schemes is similar to that of the expert opinions on the basic attributes. First, the relative weights of basic attributes are used to conduct the relative weights between expert group opinions. The preceding aggregation method is subsequently used to synthesize expert group opinions on the basic attributes to evaluate supplier collaboration configuration schemes. Similarly, the ignorance caused by the relative weights is deleted from the synthesized results. More specifically, in a similar way to Eq. (18), weights of the opinions $m_{l}^{k}$ of the experts $t_{k}$ on the supplier collaboration configuration schemes $A_{l}(1 \leq l \leq M)$ are computed as follows:

$$
w_{l}^{k}(\bar{\Omega})=\frac{\tilde{m}_{\Omega, i}^{k}}{\bar{m}_{\Omega, i}^{k}+\tilde{m}_{\Omega, i}^{k}}
$$

Combining with Eq. (20), the weight functions of expert opinions $m_{l}^{k}$ can be calculated by using the following formulae (i.e., Eqs. (29)-(31)):

$$
\begin{gathered}
\{\varnothing\}: w_{l}^{k}(\varnothing)=m_{\Omega, l}^{k} \prod_{n=1}^{N}\left(1+\frac{m_{n, l}^{k}}{m_{\Omega, l}^{k}}\right) \\
\left\{H_{n}\right\}: \quad w_{l}^{k}\left(H_{n}\right)=\frac{m_{\Omega, l}^{k}}{m_{n, l}^{k}+m_{\Omega, l}^{k}} \\
\{\bar{\Omega}\}: \quad w_{l}^{k}(\bar{\Omega})=\frac{\tilde{m}_{\Omega, i}^{k}}{\bar{m}_{\Omega, i}^{k}+\widetilde{m}_{\Omega, i}^{k}}
\end{gathered}
$$

Then, we can compute the weight functions of expert group opinions for the supplier collaboration configuration schemes $A_{l}(1 \leq l \leq M)$ according to the following formulae (i.e., Eqs. (32)-(34)):

$$
\begin{gathered}
\{\varnothing\}: w_{l}(\varnothing)=\min \left\{w_{l}^{k}(\varnothing) \mid k=1,2, \ldots, T\right\} \\
\left\{H_{n}\right\}: w_{l}\left(H_{n}\right)=\min \left\{w_{l}^{k}\left(H_{n}\right) \mid k=1,2, \ldots, T\right\} \\
\{\bar{\Omega}\}: w_{l}(\bar{\Omega})=\min \left\{w_{l}^{k}(\bar{\Omega}) \mid k=1,2, \ldots, T\right\}
\end{gathered}
$$

Hereby, the standardized belief function can be computed as follows:

$$
m_{l}=\hat{\theta} \oplus_{\varnothing \neq A \subset \Omega} A^{w_{l}(A)}
$$

where the standardized coefficient is $\hat{\theta}=\left(1-m_{l}(\varnothing)-m_{l}(\bar{\Omega})\right)^{-1} . \quad m_{l}(\bar{\Omega})$ is introduced to delete the ignorance so that it ensures the rationality of the synthesized results.

\subsection{Calculation of evaluation rating utilities}

The evaluation rating utilities of the experts are independently provided so that they meet the additive utility condition. Thus, using the weighted average method, we can compute the expert group evaluation ratings as follows:

$$
u\left(H_{\mathrm{n}}\right)=\sum_{k=1}^{T} \lambda_{k} u^{k}\left(H_{n}\right) \quad(n=1,2, \ldots, N)
$$

\subsection{Determination of the optimal supplier collaboration configuration scheme}

Different experts have different evaluation rating utilities because they have diverse experiences, types and amount of knowledge as well as levels of education. That is to say, $u^{k}\left(H_{n}\right)(k=1,2, \ldots, T)$ may be different for experts $t_{k}$. The minimum/maximum regret value $\operatorname{method}^{21}$ can be adopted to generate group orders of the supplier collaboration configuration schemes and hereby 
determine the optimal supplier collaboration configuration scheme.

\section{Group selection process of supplier collaborative configuration problems}

The group selection process of the supplier collaborative configuration problems can be summarized as follows.

\subsection{Identify experts, attributes and schemes of supplier collaborative configuration problems}

Experts from various departments (or fields) are selected by the cross-departmental comprehensive administrators of the enterprise, who recognize basic attributes under the goal of supplier collaborative configuration selection and their respective types such as benefit attributes and cost attributes. Moreover, they establish the evaluation rating set of all basic attributes. All feasible collaborative configuration schemes (or alternatives) are subsequently listed or identified. Thus, the selection problem of the supplier collaborative configuration schemes is constructed. The numbers of experts, basic attributes, and the selected schemes are denoted by $\mathrm{T}, \mathrm{L}$, and $\mathrm{M}$, respectively.

\subsection{Determine weights of the attributes and experts}

The relative weights of the basic attributes and experts can be determined according to Eq. (12)-(17) and Eq. (21)-(26) or by the relative comparison method developed by Ölcer ${ }^{21}$ or the chain ordered pair-wise comparison method proposed by $\mathrm{Li}^{33}$.

\subsection{Collect evaluations and evaluation rating utilities of experts}

The distributed evaluations of the basic attributes of each supplier collaborative configuration scheme from experts are initially collected. The evaluation rating utilities of experts are subsequently collected. Finally, the group selection model could be built with the values of the quantitative basic attributes through using the evaluation rating utilities.

\subsection{Obtain group evaluations of supplier collaborative configuration schemes}

Using the expert weights, the modified Denoeux cautiously synthesized rules are used to aggregate the evaluations of experts on the basic attributes for every supplier collaborative configuration scheme. Hereby, using the attribute weights, the modified Denoeux cautiously synthesized rules are utilized to aggregate the group evaluations of supplier collaborative configuration schemes. Thus, the group evaluations of the supplier collaborative configuration schemes are obtained according to Eq. (27).

\subsection{Estimate the aggregation utilities of evaluation ratings}

The aggregation utilities of the evaluation ratings for the supplier collaborative configuration schemes are calculated.

\subsection{Rank the supplier collaborative configuration schemes}

Based on the aggregation utilities of the evaluation ratings for collaborative configuration schemes, we can calculated their maximum, minimum, and average utilities, which are employed to generate the ranking order of the collaborative configuration schemes through using the maximum/minimum regret value method ${ }^{20}$.

\section{Case study}

Six types of new cars are currently designed and developed by an automobile manufacturing enterprise. Central collaboration model was adopted to establish a relationship with suppliers and manage them collaboratively. Let us consider an example of the instrument panel, in which 15 suppliers supply the six types of new cars. At a certain time point, the enterprise formulate four supplier collaboration configuration schemes on different suppliers and different types of car supply collaboration configuration schemes after negotiating with the purchasing, quality, and R\&D departments according to the supplier rules and regulations of the enterprise. To identify/choose the optimal supplier collaboration configuration scheme, one representative from each department should be selected to eventually form an expert group. Each supplier collaboration configuration scheme should be evaluated on the following four aspects: production capacity, R\&D capability, improvement room, and collaborative development.

Assume that the relative weight vector of the four attributes are $\left(w_{1}, w_{2}, w_{3}, w_{4}\right)=(0.1,0.3,0.3,0.3)$, the relative 
weight vector of the four experts are $\left(\lambda_{1}, \lambda_{2}, \lambda_{3}, \lambda_{4}\right)=(0.3,0.2,0.2,0.3)$, the evaluation rating set is $\Omega=\left\{H_{1}, H_{2}, H_{3}, H_{4}, H_{5}\right\}=\{$ Poor, Average, Good, Very Good, Excellent $\}$, and the evaluation rating utility sets of the four experts are $\left\{u^{1}\left(H_{1}\right)=0, u^{1}\left(H_{2}\right)=0.4, u^{1}\left(H_{3}\right)=0.6\right.$, $\left.u^{1}\left(H_{4}\right)=0.8, \quad u^{1}\left(H_{5}\right) \quad=1\right\}, \quad\left\{u^{2}\left(H_{1}\right)=0, \quad u^{2}\left(H_{2}\right)=0.3\right.$, $\left.u^{2}\left(H_{3}\right)=0.5, u^{2}\left(H_{4}\right)=0.8, u^{2}\left(H_{5}\right)=1\right\},\left\{u^{3}\left(H_{1}\right)=0, u^{3}\left(H_{2}\right)=0.5\right.$, $\left.u^{3}\left(H_{3}\right)=0.7, u^{3}\left(H_{4}\right)=0.8, u^{3}\left(H_{5}\right)=1\right\},\left\{u^{4}\left(H_{1}\right)=0, u^{4}\left(H_{2}\right)=0.5\right.$, $\left.u^{4}\left(H_{3}\right)=0.7, u^{4}\left(H_{4}\right)=0.9, u^{4}\left(H_{5}\right)=1\right\}$, respectively.

Firstly, the original evaluations of the supplier collaboration configuration schemes are independently given by the four experts, depicted as in Table 1.

In Table 1 , the evaluation $(0,0,0.8,0,0,0.2)$ of the supplier collaboration configuration scheme $A_{1}$ on the production capability given by for the expert $t_{1}$ can be represented by $\left\{\left(H_{1}, 0\right),\left(H_{2}, 0\right),\left(H_{3}, 0.8\right),\left(H_{4}, 0\right),\left(H_{5}, 0\right),(\Omega, 0.2)\right\}$. Others may be similarly explained.
Secondly, according to Eqs. (9) and (10), the modified Denoeux cautiously synthesized rules are used to aggregate the group evaluations of the supplier collaboration configuration schemes on the all four attributes, depicted as in Table 2. The aggregated evaluation of the expert group for the supplier collaboration configuration scheme $A_{1}$ on each basic attribute is complete. Meanwhile, for other three supplier collaboration configuration schemes, only the aggregated evaluations on special attributes are complete. According to the synthesized method on each expert opinion weight function of the modified Denoeux cautiously synthesized rules, only when all of the expert opinions are incomplete evaluation, the aggregated group opinion is incomplete evaluation. Compared with Table 1, the incomplete evaluations derived from Table 2 are all incomplete.

Table 1 Evaluations of the supplier collaboration configuration schemes given by the experts

\begin{tabular}{cccccc}
\hline \multirow{2}{*}{ Schemes Experts } & Production Capacity & R\&D Capability & Improvement Room & $\begin{array}{c}\text { Collaborative } \\
\text { Development }\end{array}$ \\
\hline & $t_{1}$ & $(0,0,0.8,0,0,0.2)$ & $(0,0,0.8,0,0,0.2)$ & $(0,0,0,0.8,0,0.2)$ & $(0,0,0.7,0,0,0.3)$ \\
$A_{1}$ & $t_{2}$ & $(0,0,0.7,0.1,0,0.2)$ & $(0,0,0.7,0,0,0.3)$ & $(0,0,0,0.8,0.1,0.1)$ & $(0,0,0.6,0.2,0,0.2)$ \\
& $t_{3}$ & $(0,0,0.8,0.1,0,0.1)$ & $(0,0,0.7,0.1,0,0.2)$ & $(0,0,0.2,0.7,0,0.1)$ & $(0,0,0.7,0.2,0,0.1)$ \\
& $t_{4}$ & $(0,0,0.7,0.1,0,0.2)$ & $(0,0,0.8,0.1,0,0.1)$ & $(0,0,0.2,0.7,0,0.1)$ & $(0,0,0.7,0.2,0,0.1)$ \\
\hline & $t_{1}$ & $(0,0,0,0.9,0.1,0)$ & $(0,0,0,0.3,0.7,0)$ & $(0,0,0,0.6,0,0.4)$ & $(0,0,0,0,0.9,0.1)$ \\
$A_{2}$ & $t_{2}$ & $(0,0,0.1,0.9,0,0)$ & $(0,0,0.1,0.8,0,0.1)$ & $(0,0,0.2,0.7,0,0.1)$ & $(0,0,0.7,0.1,0,0.2)$ \\
& $t_{3}$ & $(0,0,0,0.7,0.2,0.1)$ & $(0,0,0,0.9,0.1,0)$ & $(0,0,0.6,0.3,0,0.1)$ & $(0,0,0,0.8,0.2,0)$ \\
& $t_{4}$ & $(0,0,0,0.8,0.2,0)$ & $(0,0,0,0.9,0.1,0)$ & $(0,0,0.6,0.2,0,0.2)$ & $(0,0,0,0.9,0.1,0)$ \\
\hline \multirow{2}{*}{$A_{3}$} & $t_{1}$ & $(0,0,0,0.6,0,0.4)$ & $(0,0,0.3,0.7,0,0)$ & $(0,0,0,0.2,0.8,0)$ & $(0,0,0.1,0.8,0,0.1)$ \\
& $t_{2}$ & $(0,0,0.1,0.6,0,0.3)$ & $(0,0,0.1,0.7,0,0.2)$ & $(0,0,0.3,0.5,0,0.2)$ & $(0,0,0.2,0.6,0,0.2)$ \\
& $t_{3}$ & $(0,0,0.2,0.7,0,0.1)$ & $(0,0,0.2,0.8,0,0)$ & $(0,0,0,0.8,0,0.2)$ & $(0,0,0.2,0.7,0,0.1)$ \\
& $t_{4}$ & $(0,0,0.2,0.7,0,0.1)$ & $(0,0,0.3,0.6,0,0.1)$ & $(0,0,0,0.8,0.1,0.1)$ & $(0,0,0.2,0.8,0,0)$ \\
\hline \multirow{2}{*}{$A_{4}$} & $t_{1}$ & $(0,0,0.4,0.6,0,0)$ & $(0,0,0.3,0.7,0,0)$ & $(0,0,0,0.3,0.6,0.1)$ & $(0,0,0.4,0.4,0,0.2)$ \\
& $t_{2}$ & $(0,0,0.3,0.6,0,0.1)$ & $(0,0,0.2,0.6,0,0.2)$ & $(0,0,0.2,0.7,0,0.1)$ & $(0,0,0.3,0.6,0,0.1)$ \\
& $t_{3}$ & $(0,0,0.4,0.6,0,0)$ & $(0,0,0.3,0.7,0,0)$ & $(0,0,0.1,0.8,0,0.1)$ & $(0,0,0.2,0.7,0,0.1)$ \\
& $t_{4}$ & $(0,0,0.4,0.6,0,0)$ & $(0,0,0.3,0.7,0,0)$ & $(0,0,0.4,0.6,0,0)$ & $(0,0,0.2,0.6,0,0.2)$ \\
\hline
\end{tabular}

Table 2 Aggregated evaluations of the expert group for the schemes on attributes

\begin{tabular}{ccccc}
\hline Schemes & Production Capacity & R\&D Capability & Improvement Room & Collaborative Development \\
\hline$A_{1}$ & $(0,0,0.8318,0.104,0,0.0642)$ & $(0,0,0.8,0.1,0,0.1)$ & $(0,0,0.184,0.7068,0.0546,0.0546)$ & $(0,0,0.7297,0.2085,0,0.0619)$ \\
$A_{2}$ & $(0,0,0.0504,0.777,0.1726,0)$ & $(0,0,0.0343,0.5432,0.4225,0)$ & $(0,0,0.4927,0.4566,0,0.0507)$ & $(0,0,0.1807,0.4182,0.401,0)$ \\
$A_{3}$ & $(0,0,0.2085,0.7297,0,0.0619)$ & $(0,0,0.3,0.7,0,0)$ & $(0,0,0.0961,0.4425,0.4614,0)$ & $(0,0,0.2,0.8,0,0)$ \\
$A_{4}$ & $(0,0,0.4,0.6,0,0)$ & $(0,0,0.3,0.7,0,0)$ & $(0,0,0.2539,0.3809,0.3652,0)$ & $(0,0,0.3767,0.5651,0,0.0582)$ \\
\hline
\end{tabular}

The modified Denoeux cautiously synthesized rules are used to aggregate the expert group opinions on the supplier collaboration configuration schemes between different attributes and hereby obtain the expert group aggregated evaluations, depicted as in Table 3.

Table 3 Aggregated evaluations of the expert group for the supplier collaboration configuration schemes

\begin{tabular}{ccccccc}
\hline Schemes & $H_{1}$ & $H_{2}$ & $H_{3}$ & $H_{4}$ & $H_{5}$ & $\Omega$ \\
\hline$A_{1}$ & 0 & 0 & 0.5021 & 0.4521 & 0.0349 & 0.0109 \\
$A_{2}$ & 0 & 0 & 0.333 & 0.3752 & 0.2918 & 0 \\
$A_{3}$ & 0 & 0 & 0.1921 & 0.5124 & 0.2955 & 0 \\
$A_{4}$ & 0 & 0 & 0.2565 & 0.4886 & 0.2549 & 0 \\
\hline
\end{tabular}


Thirdly, according to Eq. (17), the expert group aggregated utilities of the four supplier collaboration configuration schemes are obtained as $\left\{u\left(H_{1}\right)=0\right.$, $\left.u\left(H_{2}\right)=0.43, u\left(H_{3}\right)=0.63, u\left(H_{4}\right)=0.83, u\left(H_{5}\right)=1\right\}$. Thus, we obtain the expert group aggregated evaluation expecting utilities, depicted as in Table 4.

Table 4 Expecting utility and order of the supplier collaboration configuration schemes

\begin{tabular}{ccccc}
\hline Expecting Utilities & $A_{1}$ & $A_{2}$ & $A_{3}$ & $A_{4}$ \\
\hline Minimum Values & 0.7265 & 0.813 & 0.8418 & 0.822 \\
Maximum Values & 0.7373 & 0.813 & 0.8418 & 0.822 \\
Average Vales & 0.7319 & 0.813 & 0.8418 & 0.822 \\
Ranking Order & 4 & 3 & 1 & 2 \\
\hline
\end{tabular}

Lastly, according to Table 4, using the minimum/maximum regret value method, we can calculate the maximum regret values of the expecting utilities of the four supplier collaboration configuration schemes as follows:

$R\left(A_{1}\right)=\max \{\max \{0.813,0.8418,0.822\}-0.7265,0\}=0.115$, $R\left(A_{2}\right)=\max \{\max \{0.7373,0.8418,0.822\}-0.813,0\}=0.0288$, $R\left(A_{3}\right)=\max \{\max \{0.7373,0.813,0.822\}-0.8418,0\}=0$, $R\left(A_{4}\right)=\max \{\max \{0.7373,0.813,0.8418\}-0.822,0\}=0.0198$ It is easy to see that the supplier collaboration configuration scheme $A_{3}$ has both the minimum and maximum regret values. Hence, $A_{3}$ is the optimal supplier collaboration configuration scheme among the four supplier collaboration configuration schemes. $A_{4}$ is optimal among the rest of the three supplier collaboration configuration schemes by deleting $A_{3}$ and then repeating the preceding calculation. Similarly, the optimal supplier collaboration configuration scheme $A_{2}$ can be obtained by deleting scheme $A_{4}$ and then calculating the maximum regret values of the rest of the two schemes. The ranking order of the four supplier collaboration configuration schemes is $A_{3} \succ A_{4} \succ A_{2} \succ A_{1}$. Therefore, we can determine $A_{3}$ is the optimal supplier collaboration configuration scheme.

If the selection purpose is to only determine the optimal supplier collaboration configuration scheme, then the minimum and maximum regret values can be obtained after calculating the maximum regret value of the expecting utility of each supplier collaboration configuration scheme. Generally, to enhance the reliability of the selection results and thus ensure the prompt provision of the secondary-optimal selection if the optimal selection fails to work, providing the ranking order of all supplier collaboration configuration schemes is highly recommended.

Additionally, if the aggregated evaluations of the supplier collaboration configuration schemes on the experts are complete, then the minimum, maximum and average values of the expecting utilities for supplier collaboration configuration schemes are identical. The ranking order of the supplier collaboration configuration schemes is the same as that of the average values based on the expecting utility.

\section{Conclusion}

The supplier collaborative configuration problems involve many aspects and factors such as suppliers, experts and their importance (i.e., weights), attributes and their importance (i.e., weights), and evaluation ratings and their utility. In the above, we propose the group selection model and method for solving such supplier collaborative configuration problems. The main conclusions or contributions can be summarized as follows:

(1) Determining the group optimal selection of the supplier collaborative configuration schemes based on evidential theory is rational and feasible. The distributed evaluations and utilities with incomplete information are used to accurately describe the basic attribute information. Furthermore, the developed model has several advantages/benefits. On the one hand, the developed method loosens the constraints of the independence of expert opinions, which allow experts to share their experience and knowledge so that the optimal selection process of the supplier collaborative configuration schemes may coincide more with reality. On the other hand, the difficulty of attribute selection is reduced and the dominant or recessive correlation between partial attributes cannot be repelled by loosening the independence requirement. Thus, the robustness of the optimal selection results of the supplier collaborative configuration schemes are enhanced.

(2) The traditional Denoeux cautious combination rules are inapplicable due to the ignorance caused by the relative weights. However, the modified Denoeux cautious combination rules can be used to solve the above problem by expelling such ignorance from the aggregation process of expert opinions and hereby deleting the ignorance from the combination results. 
(3) The results of the case study indicate that the developed group selection method for supplier collaborative configuration problems with correlation of experts and attributes is rational, feasible and applicable.

\section{References}

1. J. K. Shank and V. Govindarajan, Strategic Cost Management (The Free Press, NewYork, 1993).

2. R.Cooper and R. Slagmulder, Inter-organizational cost management and relational context, Accounting, Organizations and Society 29(1) (2004) 1-26.

3. A. F. Coad and J. Cullen, Inter-organizational cost management: towards an evolutionary perspective, Management Accounting Research 17(4) (2006) 342-369.

4. Y. Fu, Cost synergy management in e-supply chain, Pacific-Asia Management Accounting 2(1) (2007) 35-53.

5. C. M. Su, D. J. Horng and M. L. Tseng, Improving sustainable supply chain management using a novel hierarchical grey-DEMATEL approach, Journal of Cleaner Production (5) (2015) 1-13.

6. C. N. Verdouw, A. J. M. Beulens and H. A. Reijers, A control model for object virtualization in supply chain management, Computers in Industry 68 (2015) 116-131.

7. D. J. F. Jeng, Generating a causal model of supply chain collaboration using the fuzzy DEMATEL technique, Computers \& Industrial Engineering 87 (2015) 283-295.

8. S. Y. P. Lu, H. Y. K. Lau and C. K. F. Yiu, A hybrid solution to collaborative decision-making in a decentralized supply-chain, Journal of Engineering and Technology Management 29(1) (2012) 95-111.

9. Y. H. Fu, H. G. Zhang and J. F. Tang, Supplier performance evaluation and decision based on evidential reasoning, Journal of Northeast University 30(11) (2009) 1546-1549.

10. C. T. Chen, C. T. Lin and S. F. Huang, A fuzzy approach for supplier evaluation and selection in supply chain management, International Journal of Production Economics 102(2) (2006) 289-301.

11. T. Y. Wang and Y. H.Yang, A fuzzy model for supplier selection in quantity discount environments, Expert Systems with Applications 36(10) (2009) 12179-12187.

12. D. D. Wu, Y. D. Zhang, D. X. Wu and D. L. Olson, Fuzzy multi-objective programming for supplier selection and risk modeling: A possibility approach, European Journal of Operational Research 200(3) (2010) 774-787.

13. V. Jayaraman, R. Srivastava and W. C. Benton, Supplier Selection and Order Quantity Allocation: A Comprehensive Model, Journal of Supply Chain Management 35(2) (1999) 50-58.

14. D. L. Xu and J. B. Yang, Intelligent decision system based on the evidential reasoning approach and its applications, Journal of Telecommunications and Information Technology 3 (2005) 73-80.
15. J. B. Yang and S. Pratyush, Multiple attribute design evaluation of complex engineering products using the evidential reasoning approach, Journal of Engineering Design 8(3) (1997) 211-230.

16. M. Sonmez, J. B. Yang and G. D. Holt, Addressing the contractor selection problem using an evidential reasoning approach, Engineering, Construction and Architectural Management 8(3) (2001) 198-210.

17. J. B. Yang, Rule and utility based evidential reasoning approach for multiattribute decision analysis under uncertainties, European Journal of Operational Research 131(1) (2001) 31-61.

18. J. B. Yang and D. L. Xu, On the evidential reasoning algorithm for multiple attribute decision analysis under uncertainty, IEEE Transactions on Systems, Man, and Cybernetics 32(3) (2002) 289-304.

19. D. L. Xu, J. B. Yang and Y. M. Wang, The evidential reasoning approach for multi-attribute decision analysis under interval uncertainty, European Journal of Operational Research 174(3) (2006) 1914-1943.

20. Y. M. Wang, J. B. Yang, D. L. Xu and K. S. Chin, The evidential reasoning approach for multiple attribute decision analysis using interval belief degrees, European Journal of Operational Research 175(1) (2006) 35-66.

21. A. I. Ölcer and A. Y. Odabasi, A new fuzzy multiple attribute goup decision making methodology and its applicaton to propulsion/manoeuvring system selection problem, European Journal of Operational Research 166 (2005) 93-114.

22. J. X. Fu and Y. L. Fu, An adaptive multi-agent system for cost collaborative management in supply chains, Engineering Applications of Artificial Intelligence 44 (2015) 91-100.

23. J. X. Fu and Y. L. Fu, Case-Based reasoning and multi-agents for cost collaborative management in supply chain, Procedia Engineering 29 (2012) 1088-1098.

24. G. W. Dickson, An analysis of vendor selection system and decision, Journal of Purchasing 2 (1966) 5-17.

25. E. A. Demirtas and O. Üstün, An integrated multiobjective decision making process for supplier selection and order allocation, Omega 36 (2008) 76-90.

26. D. F. Li and S. P. Wan, A fuzzy inhomogenous multiattribute group decision making approach to solve outsourcing provider selection problems, Knowledge-Based Systems 67 (2014) 71-89.

27. Z. Hu, C. J. Rao, Y. Zheng and D. Huang, Optimization decision of supplier selection in green procurement under the mode of low carbon economy, International Journal of Computational Intelligence Systems 8(3) (2015) 407-421.

28. H. X. Zhang, Y. Deng and F. T. S. Chan, A modified multi-criterion optimization genetic algorithm for order distribution in collaborative supply chain, Applied Mathematical Modelling 37(14-15) (2013) 7855-7864.

29. T. Denoeux, Conjunctive and disjunctive combination of belief functions induced by non distinct bodies of evidence, 
Artificial Intelligence 172 (2-3) (2008) 234-264.

30. M. M. Masson and T. Denoeux, Ensemble clustering in the belief functions framework, International Journal of Approximate Reasoning 52 (2011) 92-109.

31. J. B. Yang, Y. M. Wang and D. L. Xu, The evidential reasoning approach for MADA under both probabilistic and fuzzy uncertainties, European Journal of Operational Research 171 (2006) 309-343.
32. S. L. Yang, S. Ding and C. Fu, A software trust worthness evaluation model considering correlation of information sources, Chinese Journal of Management Science 17(6) (2009) 163-169.

33. D. F. Li, An approach to fuzzy multiattribute decision making under uncertainty, Information Sciences 169(1-2) (2005) 97-112. 